\title{
ORDERED RELIABILITY BITS GUESSING RANDOM ADDITIVE NOISE DECODING
}

\author{
Ken R. Duffy \\ Hamilton Institute \\ Maynooth University, Ireland \\ ken.duffy@mu.ie
}

\begin{abstract}
Modern applications are driving demand for ultrareliable low-latency communications, rekindling interest in the performance of short, high-rate error correcting codes. To that end, here we introduce a soft-detection variant of Guessing Random Additive Noise Decoding (GRAND) called Ordered Reliability Bits GRAND that can decode any moderate redundancy block-code. For a code of $n$ bits, it avails of no more than $\left\lceil\log _{2}(n)\right\rceil$ bits of code-book-independent quantized soft detection information per received bit to determine an accurate decoding while retaining the original algorithm's suitability for a highly parallelized implementation in hardware. ORBGRAND is shown to provide similar block error performance for codes of distinct classes (BCH, CA-Polar and RLC) with low complexity, while providing better block error rate performance than CA-SCL, a state of the art soft detection CA-Polar decoder.
\end{abstract}

Index Terms-URLLC, GRAND, Soft Decision, Quantization

\section{INTRODUCTION}

Maximum Likelihood (ML) decoding has been known to be optimally accurate for uniform sources since Shannon's pioneering work [1], which also established that the highest code-rates that a channel can support are achieved when the code is long. Since 1978, however, it has been known that ML decoding of linear codes is an NP-complete problem [2]. Taken together, these results have driven the practical paradigm of co-designing restricted classes of linear code-books in tandem with code-specific decoding methods that exploit the codestructure to enable computationally efficient approximateML decoding. For example, Bose-Chaudhuri-Hocquenghem (BCH) codes with hard detection Berlekamp-Massey decoding [3], [4], or CRC-Assisted Polar (CA-Polar) codes with soft detection CRC-Assisted Successive Cancellation List (CASCL) decoding [5], [6], [7], [8].

Recent applications, including augmented and virtual reality, vehicle-to-vehicle communications, the Internet of Things, and machine-type communications, have driven demand for Ultra-Reliable Low-Latency Communication (URLLC) [9], [10], [11], [12], [13]. As realizing these technologies requires short, high-rate codes, the complexity issues associated with long codes will be vacated in URLLC and so it offers the opportunity to revisit the possibility of creating high-accuracy universal decoders that are suitable for hardware implementation. In turn, the development of practical universal decoders opens up a massively larger palette of potential code-books for decoding with a single algorithmic instantiation.
Inputs: Code-book membership function $C:\{0,1\}^{n} \mapsto$ $\{0,1\}$; demodulated bits $y^{n}$; optional information $\Phi$.

Output: Decoding $c^{*, n}$, soft output $D$

$d \leftarrow 0, D \leftarrow 0$.

while $\mathrm{d}=0$ do

$z^{n} \leftarrow$ next most likely binary noise effect sequence (which may depend on $\Phi$ )

$D \leftarrow D+1$

if $C\left(y^{n} \ominus z^{n}\right)=1$ then

$c^{*, n} \leftarrow y^{n} \ominus z^{n}$

$d \leftarrow 1$

return $c^{*, n}, d, D$

end if

end while

Fig. 1. Guessing Random Additive Noise Decoding. Inputs: a demodulated channel output $y^{n}$; a code-book membership function such that $C\left(y^{n}\right)=1$ if and only if $y^{n}$ is in the code-book; and optional statistical noise characteristics or soft information, $\Phi$. Output: decoded element $c^{*, n}$; and the number of codebook queries made, $D$, a measure of confidence in the decoding.

Universal soft detection approximate-ML decoders for binary linear codes have been proposed that work on a listdecoding principle [14], [15], [16], [17], [18], [19]. In the Orders Statistics Decoding approach, rather than compute the conditional likelihood of the received signal for all members of the code-book, instead the computation is done for a restricted list of code-words that is hoped to contain the transmitted one. The algorithm permutes the columns of the parity check matrix in a manner that depends on the received signal and Gaussian elimination is performed to rewrite the code in systematic format - subject to checking to ensure a basis is identified and the real-valued computation of conditional probabilities, all of which make it a challenge to implement in hardware.

A recent alternate approach that lends itself to implementation in circuits is Guessing Random Additive Noise Decoding (GRAND) [20], [21]. It is a universal ML decoding algorithm that is suitable for use with any moderate redundancy blockcode construction in any field, including unstructured codebooks stored in a dictionary. All GRAND algorithms work by taking the demodulated received sequence and querying if it is in the code-book. If it is in the code-book, it is the decoding. If it is not in the code-book, then the most likely non-zero binary noise sequence is subtracted from the received sequence and 
what remains is queried for membership of the code-book. This process proceeds until an element of the code-book is identified, which is the decoding. Pseudo-code for GRAND can be found in Fig. 1. Where GRAND algorithmic variants differ is in their order of querying putative noise effects. When putative noise effects are ordered in decreasing likelihood from a noise model matched to the channel, it provably produces an ML decoding even for channels with memory in the absence of interleaving [21], [22], [23]. The original algorithm assumed the decoder obtained only hard decision demodulated symbols from the receiver. The simplicity of its operation and the evident parallelizability of code-book querying has resulted in the proposal of an efficient circuit implementation [24].

Incorporating per-realization soft detection information into decoding decisions is known to improve accuracy [25], [26], [27]. Doing so, however, requires that additional information be passed from the receiver to the decoder, which typically necessitates its quantization for efficient transmission. Symbol Reliability GRAND (SRGRAND) [28], [29], [30] uses the most extreme quantized soft information where one additional bit tags each demodulated symbol as being reliably or unreliably received. Implementing SRGRAND in hardware retains the desirable parallelizability and is no more challenging than doing so for GRAND, but symbol reliability information does not exploit the full benefit of soft information.

At the other extreme, Soft GRAND (SGRAND) [23] uses one real-valued piece of soft information per demodulated symbol to build a distinct noise-effect query order for each received signal, resulting in soft detection ML decodings. Its execution is, however, algorithmically involved. Using dynamic max-heap data structures it is possible to create a semi-parallelizable software implementation, but the design does not lend itself to realization in hardware.

Here we introduce Ordered Reliability Bits GRAND (ORBGRAND), which aims to bridge that gap between SRGRAND and SGRAND by obtaining the decoding accuracy of the latter in an algorithm that is entirely parallelizable and suitable for implementation in circuits. It avails of a code-book-independent quantization of soft information: the decreasing rank order of the reliability of each bit in a received block. It uses this to map a fixed, pre-determined, series of putative noise queries to their appropriate locations in the received block. If the a posteriori bit flip probabilities match a member of a broad parametric class of models it provides ML decodings, and approximate-ML decodings otherwise.

\section{ORDERED RELIABILITY Bits GRAND}

For a block-code of length $n$ bits, all GRAND variants require a code-book membership function $C:\{0,1\}^{n} \mapsto\{0,1\}$ that, given a binary string of length $n$, returns 1 if the string is in the code-book and 0 otherwise. For a linear code in any field size, establishing code-book membership can be achieved with a single matrix multiplication. For a CRC code, the computation is even simpler [31]. For an unstructured code, checking code-book membership can be performed by a dictionary look-up.
Assume that binary code-words, $x^{n} \in\{0,1\}^{n}$, are impacted by independent continuous additive noise resulting in a random received signal. Let $y^{n}$ denote its hard decision demodulation. ORBGRAND provides a decoding based on the hard decision demodulation, $y^{n}$, and a soft-information informed vector that records the rank ordering of the reliability of the received bits from least reliable to most reliable, $r^{n}$, which is a permutation of $(1, \ldots, n)$. ORBGRAND does not require any further information about the received signal or the channel. As it attempts to identify the effect of the noise that has impacted the communication, key to its performance is its order of querying putative noise effect sequences.

The algorithmic premise is to create a pre-determined rank ordered list of base noise effect sequences, $z^{n, 1}, z^{n, 2}, \ldots$ with the assumption that the first bit is the least reliable, the second bit is the second least reliable, and so forth. For a given received block $y^{n}$ with rank ordered bit reliabilities described by the permutation $r^{n}$, ORBGRAND maps bits in these base sequences to the correct location via the permutation encoded in $r^{n}$. That is, ORBGRAND queries the sequences $(z \circ r)^{n, 1},(z \circ r)^{n, 2}, \ldots$, where $(z \circ r)^{n, i}=\left(z_{r_{1}}^{i}, \ldots, z_{r_{n}}^{i}\right)$. Thus to complete the description of the algorithm, we need only determine the ordered base sequences $z^{n, 1}, z^{n, 2}, \ldots$ that would be used if $r^{n}=(1,2, \ldots, n)$ corresponding to bits in the received block having reliability that increases with index.

If only hard detection information was provided and the channel was a binary symmetric channel, to generate putative noise sequences in order of increasing likelihood it is sufficient to order them in increasing Hamming weight breaking ties arbitrarily: if $w_{\mathrm{H}}\left(z^{n, i}\right)<w_{\mathrm{H}}\left(z^{n, j}\right)$, where $w_{\mathrm{H}}\left(z^{n}\right)=\sum_{k=1}^{n} z_{k}^{n}$ is the number of ones in the sequence, then $z^{n, i}$ would be queried before $z^{n, j}$. Akin to that, the observation underlying ORBGRAND is that if the rank ordered a posteriori reliability of bits follow a function from a broad parametric class described in Section II, to rank order putative noise sequences in increasing likelihood, it is sufficient to order them by what we call the Logistic Weight:

$$
w_{\mathrm{L}}\left(z^{n}\right)=\sum_{k=1}^{n} k 1_{\left\{z_{k}^{n}=1\right\}} .
$$

Thus a putative noise effect sequence in increasing order of bit reliability is assigned a weight that is not the number of bits flipped, but the sum of the indices of flipped bits.

In the language of combinatorics, to determine the base sequences $\left\{z^{n, i}\right\}$ requires a method to generate all integer partitions of $w_{\mathrm{L}}=0,1,2, \ldots, n(n+1) / 2$ with unique parts and no part being larger than $n$. Algorithms have been described in the literature [32] that can generate these in real-time or offline for storage, and the upper panel of Fig. 2 illustrates the first 100 ORBGRAND queries, with bit positions assumed to be in decreasing order of reliability. The first sequence corresponds to no bits being flipped, which has $w_{\mathrm{L}}=0$. The second query corresponds to only the most unreliable bit being flipped, having $w_{\mathrm{L}}=1$. The third corresponds to only the second most unreliable bit being flipped, which has $w_{\mathrm{L}}=2$. The next query is either the noise effect where only the third most unreliable 


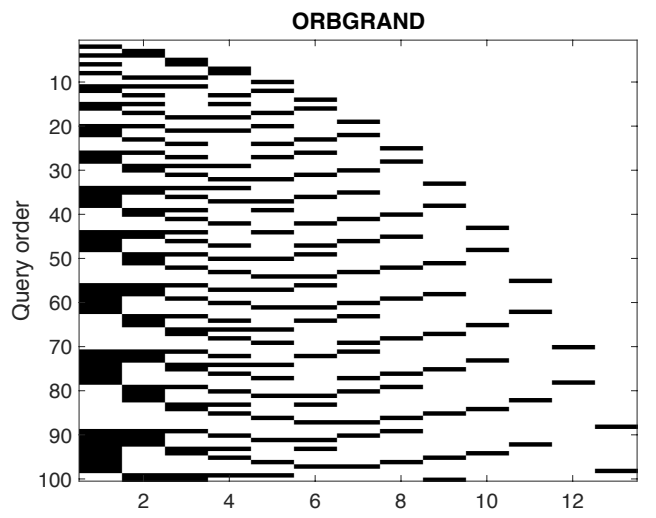

Fig. 2. First 100 ORBGRAND noise effect queries where bit positions are in decreasing order of a posteriori bit flip likelihood. Each row is a noise sequence with white being no bit flip and black corresponding to a bit flip.

bit is flipped, which has $w_{\mathrm{L}}=3$, or the one where first and second most unreliable bits are flipped, which also has $w_{\mathrm{L}}=3$, and so this tie is broken arbitrarily. The ordering proceeds in that fashion. While this fully describes the algorithm, it does not justify why it works.

For a block of length $n$ bits and one use of the channel, assume that the a posteriori bit flip probabilities, in $[0,1 / 2)$, are $A_{1}, \ldots, A_{n}$ and that, rank ordered from highest (i.e. least reliable) to lowest they are $A_{1, n} \geq A_{2, n} \geq \cdots \geq A_{n, n}$. If the latter were consistent with a model such that the $a$ posteriori likelihood that the $i^{\text {th }}$ least reliable hard demodulated bit $i$ was flipped was

$$
A_{n, i}=\frac{2^{-\beta i}}{1+2^{-\beta i}}
$$

for some $\beta>0$, then the probability of the base putative error sequence $z^{n}$ that has only the bits $i_{1}, \cdots, i_{K}$ flipped would be

$$
\prod_{i=1}^{n}\left(1-A_{n, i}\right) \prod_{j=1}^{K} \frac{A_{n, i_{j}}}{1-A_{n, i_{j}}} \propto \prod_{j=1}^{K} \frac{A_{n, i_{j}}}{1-A_{n, i_{j}}}=2^{-\beta w_{\mathrm{L}}\left(z^{n}\right)} .
$$

Thus, in terms of noise sequence likelihood, to compare the rank-order of two base putative error sequences, one need only evaluate their logistic weights, $w_{\mathrm{L}}\left(z^{n}\right)=\sum_{j=1}^{K} i_{j}$, and order those. As a result, if for a given realization Eq. (1) holds for any $\beta$, then ORBGRAND provides a soft ML decoding.

The richness of the class of functions in Eq. (1) is central to ORBGRAND's universality. Comparisons of the rank ordered a posteriori likelihoods of bit flip from an AWGN channel and the best-fit $\beta$ for the logistic model as determined by regression is shown in Fig. 3, where good agreement is found. Note that the crucial matter is not how perfect the fit is, but whether it is good enough to produce rank-ordered noise sequences so that the guessing order is approximately ML.

\section{Performance eVAluation}

ORBGRAND can decode any block-code. For illustration we consider three classes of binary linear $[n, k]$ codes: $\mathrm{BCH}$ codes, requiring $n$ to be a power of two minus one, for which there is an accurate hard-detection decoder; CA-Polar codes,

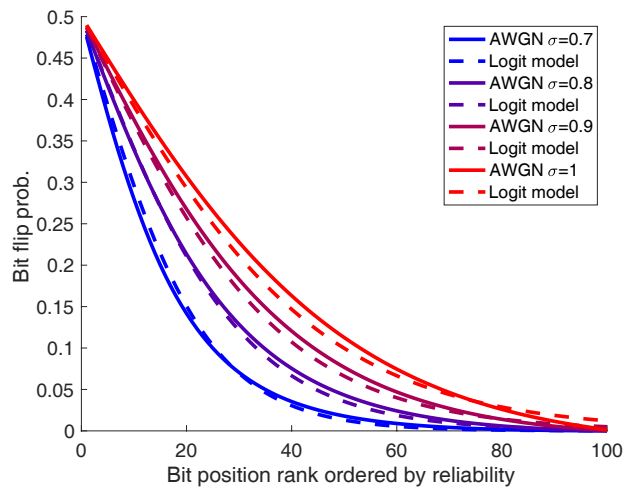

Fig. 3. Comparing a posteriori rank ordered bit flip probabilities from a BPSK AWGN channel and the class of functions used by ORBGRAND.
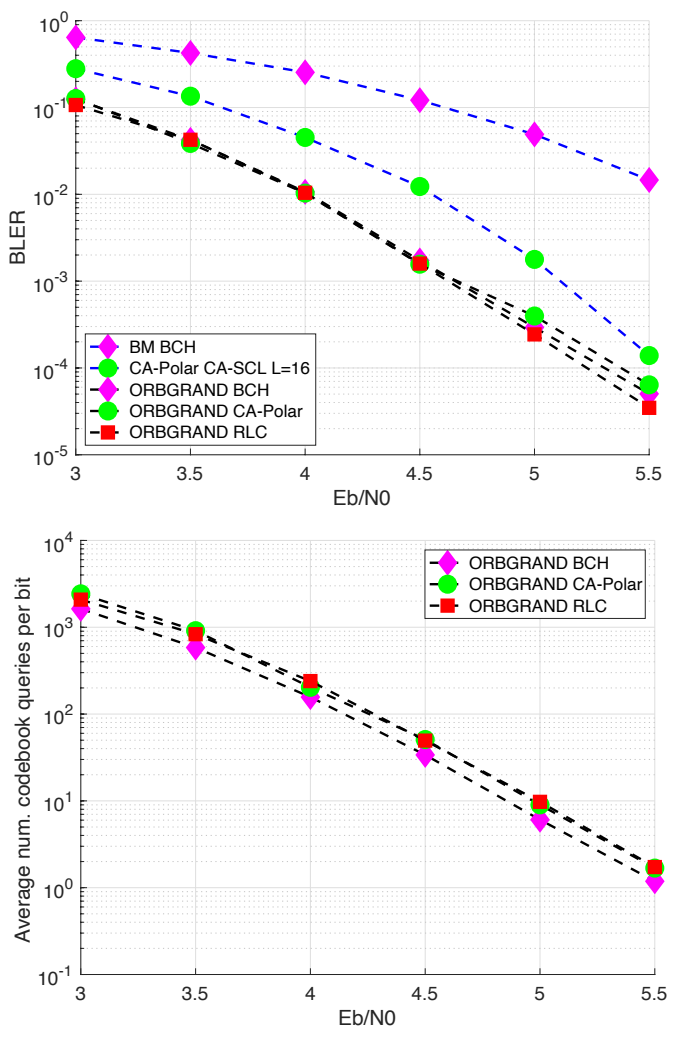

Fig. 4. Rate $0.83 \mathrm{BCH}(127,106)$, and rate $0.82 \mathrm{CA}-\mathrm{Polar}$ and $\mathrm{RLC}(128,105)$ in an AWGN BPSK channel. Decoding BCH with hard detection BerlekampMassey, CA-Polar with soft detection CA-SCL (list size $L=16$ ) and all three with ORBGRAND. Upper panel: BLER vs Eb/N0. Lower panel: average number of code-book queries per bit until a decoding is identified vs Eb/N0.

which will used for all control channel communications in $5 \mathrm{G}$ NR and require $n$ to be a power of two, for which there is a dedicated soft-detection decoder, CA-SCL; and Random Linear Codes (RLCs), which includes all linear codes and exists for all $[n, k]$ pairs. RLCs are been theoretically known to be high-performing [33], [34], but the lack of an efficient universal decoder means their practical potential has been little explored.

The upper panel of Fig. 4 provides a block error rate 

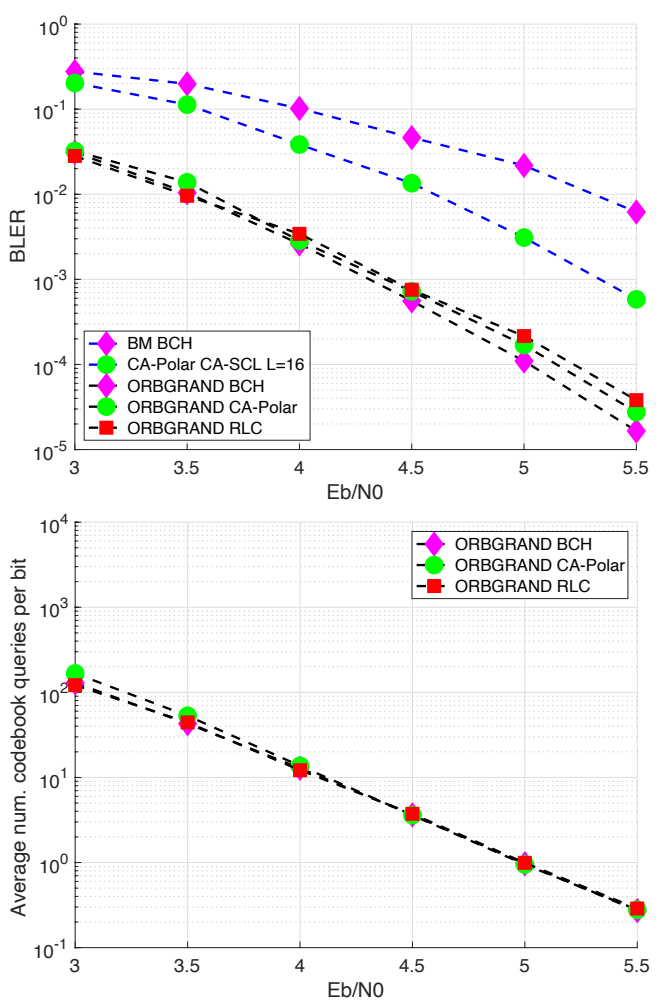

Fig. 5. As in Fig. 4, but for rate $0.71 \mathrm{BCH}(63,45)$, and rate $0.72 \mathrm{CA}-$ Polar and $\operatorname{RLC}(64,44)$.

(BLER) performance comparison in an AWGN channel using BPSK. Unsurprisingly, ORBGRAND's soft detection decoding of $\operatorname{BCH}(127,106)$ significantly outperforms the hard detection BM decoding. Perhaps surprisingly, ORBGRAND's decoding of the CA-Polar $(128,106)$ is also a half $\mathrm{dB}$ better than CASCL, the dedicated CA-Polar decoder. This arises as CA-Polar codes are essentially the product of two binary linear codes: a CRC and a Polar code. In the 5G NR standard, the CRC is either 11 or 23 bits. Here CA-SCL uses 12 Polar bits for error correction to create a list of candidate code-words, and the 11 CRC bits to determine selection from that list. ORBGRAND sees the CA-Polar code as a single code and can make direct use of all 23 bits for error correction.

Also shown is the performance of RLCs of the same rate as the CA-Polar code when decoded with ORBGRAND. The performance of RLCs is effectively indistinguishable from that of the $\mathrm{BCH}$ or CA-Polar code. This indicates that either most randomly selected codes are as good as $\mathrm{BCH}$ or CA-Polar codes or there is variability, in which case there are individual codes that have better performance than the CA-Polar codes that will be used in 5G NR. While there are $35 \mathrm{BCH}$ codes with length 127 or smaller, and, when an 11 bit CRC is employed, 196 CA-Polar codes with length 128 or smaller, there are $2^{8256}$ codes available of length 128 or smaller and ORBGRAND can decode all of them.

The lower panel of Fig. 4 reports the average number of code-book queries per bit until a decoding is found by
ORBGRAND, serving as a proxy for algorithmic complexity, though code-book queries would be readily parallelized in hardware so that many are made per cycle [24]. As expected from theoretical understanding of GRAND algorithms [21], [29], complexity is essentially code-book independent. It is minimal, with about $10^{2}$ queries per bit or less for typical operational settings where a BLER of $10^{-3}$ or lower is desired.

As in Fig. 4, Fig. 5 provides a performance evaluation of ORBGRAND when used with shorter $n=64$ bit codes. Again, by making use of the 11 bit CRC for error correction rather than just detection, ORBGRAND sees a $1 \mathrm{~dB}$ gain in performance over CA-SCL and its BLER performance is essentially code-book independent. For these shorter codes, complexity is reduced further with an average of less than 5 code-book queries per bit in standard operational regimes.

The data in Figs 4 and 5 suggest that RLCs offer similar BLER performance to well-known structured code-books. This is further substantiated in Fig. 6 which, for an energy per transmitted bit of $4.5 \mathrm{~dB}$, plots BLER versus the average number of ORBGRAND code-book queries per transmitted bit. Shown is the performance of 500 individual codes when compared with the re-randomized RLC, and the CA-Polar code to be used in 5G NR. $22 \%$ of the RLCs provide better BLER than the CA-Polar code, and 50\% give BLER that is within $10^{-4}$ of it. For reference the CA-SCL BLER result is also plotted.

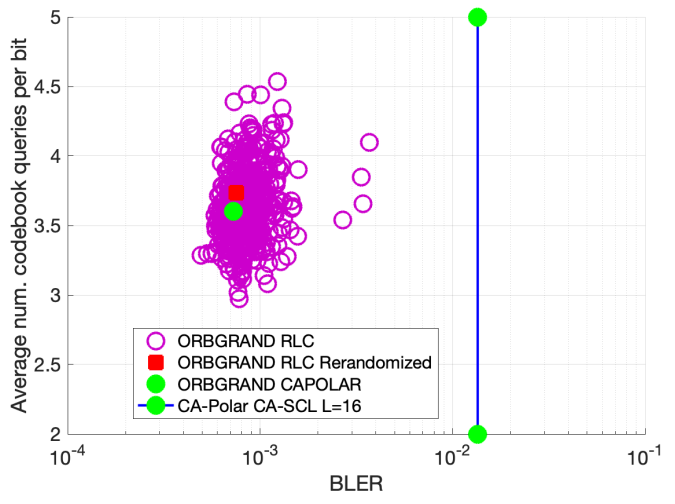

Fig. 6. BLER vs average number of code-book queries per bit for ORBGRAND with rate $0.71[64,46]$ codes at $E b / N 0=4.5 \mathrm{~dB}$. Each purple circle is one of 500 RLCs. The red square is for RLCs that are re-randomization at each communication. The green circle is the CA-Polar code. The vertical line is CA-SCL with a list size of 16 , for which the y-value has no meaning.

\section{SUMMARY}

We have introduced ORBGRAND, a universal block decoding algorithm that uses a code-book-independent quantization of soft information to inform its decoding. It is suitable for hardware implementation, and results indicate it can provide better BLER performance for moderate redundancy $5 \mathrm{G}$ NR CA-Polar codes than a state-of-the-art soft detection decoder. It is also shown that Random Linear Codes offer essentially the same performance as highly structured $\mathrm{BCH}$ and CA-Polar codes, opening up new application possibilities. 


\section{REFERENCES}

[1] C. E. Shannon, "A Mathematical Theory of Communication,” Bell Syst. Tech. J., vol. 27, pp. 379-423, 623-656, 1948.

[2] E. Berlekamp, R. McEliece, and H. Van Tilborg, "On the inherent intractability of certain coding problems (corresp.)," IEEE Trans. Inf. Theory, vol. 24, no. 3, pp. 384-386, 1978.

[3] E. Berlekamp, Algebraic coding theory. World Scientific, 1968.

[4] J. Massey, "Shift-register synthesis and BCH decoding," IEEE Trans. Inf Theory, vol. 15, no. 1, pp. 122-127, 1969.

[5] K. Niu and K. Chen, "CRC-aided decoding of Polar codes," IEEE Commun. Letters, vol. 16, no. 10, pp. 1668-1671, 2012.

[6] I. Tal and A. Vardy, "List decoding of Polar codes," IEEE Trans. Inf. Theory, vol. 61, no. 5, pp. 2213-2226, 2015.

[7] A. Balatsoukas-Stimming, M. B. Parizi, and A. Burg, "LLR-based successive cancellation list decoding of Polar codes," IEEE Trans. Signal Process., vol. 63, no. 19, pp. 5165-5179, 2015.

[8] M. Leonardon, A. Cassagne, C. Leroux, C. Jego, L.-P. Hamelin, and Y. Savaria, "Fast and flexible software polar list decoders," J. Signal Process. Syst., pp. 1-16, 2019.

[9] G. Durisi, T. Koch, and P. Popovski, "Toward massive, ultrareliable, and low-latency wireless communication with short packets," Proc. IEEE, vol. 104, no. 9, pp. 1711-1726, 2016.

[10] C. She, C. Yang, and T. Q. Quek, "Radio resource management for ultra-reliable and low-latency communications," IEEE Commun. Mag., vol. 55, no. 6, pp. 72-78, 2017.

[11] H. Chen, R. Abbas, P. Cheng, M. Shirvanimoghaddam, W. Hardjawana, W. Bao, Y. Li, and B. Vucetic, "Ultra-reliable low latency cellular networks: Use cases, challenges and approaches," IEEE Commun. Mag., vol. 56, no. 12, pp. 119-125, 2018.

[12] I. Parvez, A. Rahmati, I. Guvenc, A. I. Sarwat, and H. Dai, "A survey on low latency towards 5G: RAN, core network and caching solutions," IEEE Commun. Surv., vol. 20, no. 4, pp. 3098-3130, 2018.

[13] M. Médard, "Is 5 just what comes after 4?" Nature Electronics, vol. 3, no. 1, pp. 2-4, 2020.

[14] B. Dorsch, "A decoding algorithm for binary block codes and J-ary output channels (corresp.)," IEEE Trans. Inf. Theory, vol. 20, no. 3, pp. 391-394, 1974

[15] M. P. C. Fossorier and S. Lin, "Soft-decision decoding of linear block codes based on ordered statistics," IEEE Trans. Inf. Theory, vol. 41, no. 5, pp. 1379-1396, 1995.

[16] D. Gazelle and J. Snyders, "Reliability-based code-search algorithms for maximum-likelihood decoding of block codes," IEEE Tran. Inf. Theory, vol. 43, no. 1, pp. 239-249, 1997.

[17] A. Valembois and M. Fossorier, "Box and match techniques applied to soft-decision decoding," IEEE Trans. Inf. Theory, vol. 50, no. 5, pp. 796-810, 2004.

[18] Y. Wu and C. N. Hadjicostis, "Soft-decision decoding of linear block codes using preprocessing and diversification," IEEE Trans. Inf. Theory, vol. 53, no. 1, pp. 378-393, 2006.

[19] M. Baldi, N. Maturo, E. Paolini, and F. Chiaraluce, "On the use of ordered statistics decoders for low-density parity-check codes in space telecommand links," EURASIP J Wirel. Comm., no. 1, p. 272, 2016.

[20] K. R. Duffy, J. Li, and M. Médard, "Guessing noise, not code-words," in IEEE Int. Symp. on Inf. Theory, 2018

[21] — "Capacity-achieving guessing random additive noise decoding (GRAND)," IEEE Trans. Inf. Theory, vol. 65, no. 7, pp. 4023-4040, 2019.

[22] W. An, M. Médard, and K. R. Duffy, "Keep the bursts and ditch the interleavers," in IEEE GLOBECOMM, 2020.

[23] A. Solomon, K. R. Duffy, and M. Médard, "Soft maximum likelihood decoding using GRAND," in IEEE Int. Commun. Conf., 2020.

[24] S. M. Abbas, T. Tonnellier, F. Ercan, and W. J. Gross, "High-throughput VLSI architecture for GRAND," in IEEE SiPS, 2020

[25] A. B. Cooper, "Soft decision decoding of block codes," U.S. Army Laboratory Command, Maryland, Tech. Rep., 1988.

[26] T. Kaneko, T. Nishijima, and S. Hirasawa, "An improvement of softdecision maximum-likelihood decoding algorithm using hard-decision bounded-distance decoding," IEEE Tran. Inf. Theory, vol. 43, no. 4, pp. 1314-1319, 1997.

[27] V. Guruswami and M. Sudan, "Improved decoding of Reed-Solomon and algebraic-geometry codes," IEEE Tran. Inf. Theory, vol. 45, no. 6, pp. 1757-1767, 1999
[28] K. R. Duffy and M. Médard, "Guessing random additive noise decoding with soft detection symbol reliability information," in IEEE Int. Symp. on Inf. Theory, 2019.

[29] K. R. Duffy, M. Médard, and W. An, "Guessing random additive noise decoding with symbol reliability information (SRGRAND)," arXiv:1902.03796, Tech. Rep., 2019.

[30] K. R. Duffy, A. Solomon, K. M. Konwar, and M. Médard, "5G NR CAPolar maximum likelihood decoding by GRAND," in Annual Conference on Information Sciences and Systems, 2020.

[31] W. An, M. Médard, and K. R. Duffy, "CRC codes as error correcting codes," in IEEE ICC, 2021

[32] D. Rasmussen, C. D. Savage, and D. B. West, "Gray code enumeration of families of integer partitions," J. Comb. Theory A, vol. 70, no. 2, pp 201-229, 1995.

[33] R. G. Gallager, Information Theory and Reliable Communication. NY, USA: John Wiley \& Sons, Inc., 1968.

[34] J. T. Coffey and R. M. Goodman, "Any code of which we cannot think is good," IEEE Trans. Inf. Theory, vol. 36, no. 6, pp. 1453-1461, 1990. 